International Journal of Modern Physics C

(C) World Scientific Publishing Company

\title{
PRECISE DETERMINATION OF THE CONDUCTIVITY EXPONENT OF 3D PERCOLATION USING "PERCOLA"
}

\author{
JEAN-MARIE NORMAND ${ }^{1}$ and HANS J. HERRMANN ${ }^{2}$ \\ 1 CEA, Service de Physique Théorique, CE-Saclay \\ F-91191 Gif-sur-Yvette Cedex, FRANCE \\ 2 P.M.M.H., E.S.P.C.I., 10 rue Vauquelin \\ 75231 Paris Cedex 05, France
}

Using 20 months of CPU time on our special purpose computer "Percola" we determined the exponent for the normal conductivity at the threshold of threedimensional site and bond percolation. The extrapolation analysis taking into account the first correction to scaling gives a value of $t / \nu=2.26 \pm 0.04$ and a correction exponent $\omega$ around 1.4 .

\section{Introduction}

Percolation ${ }^{[1]}$ is the simplest model for a random medium and is applied to describe porous media, gelation, mixtures of electrical conductors, and many other disorder dominated situations. It is defined on a lattice by occupying with probability $p$ randomly and independently either the sites or the bonds with conductors. If one considers that locally current can only flow between conductors that are nearest neighbors, then only above a critical value $p_{c}$ a global current can flow through the system. The global electrical conductivity $\Sigma$ rises like

$$
\Sigma \sim\left(p-p_{c}\right)^{t}
$$

and the accurate determination of $t$, or more precisely of $t / \nu$ where $\nu$ is the exponent of the correlation length, is the aim of the present paper.

In the ' $80 \mathrm{~s}$, after a heated controversy the critical exponents for the conductivity of two-dimensional percolation were determined quite accurately ${ }^{[2,3]}$ and various conjectures ${ }^{[4]}$ could be ruled out. The three-dimensional situation remained less clear because of the larger error bars. Only recently new efforts have been made ${ }^{[5,6]}$ to obtain more precise values for $t / \nu$ in three dimensions using considerably larger 
computational means. Gingold and Lobb ${ }^{[5]}$ find $t / \nu=2.276 \pm 0.012$ and Batrouni et al. ${ }^{[6]}$ claim $t / \nu=2.282 \pm 0.005$.

Using the specially constructed processor "Percola" [7], assembled in Saclay by J.-M. Normand and M. Hajjar between 1984 and 1987, we did exact calculations on narrow bars of random conductors ("strip-bar method"), a technique that has given very precise values in two dimensions ${ }^{[2]}$ and for the superconducting-conducting mixture in three dimensions ${ }^{[8]}$. For the three-dimensional conductivity problem treated in this paper this method a dozen years ago gave $t / \nu=2.2 \pm 0.1$, however, with much smaller computational investment ${ }^{[9]}$.

In the following section we describe the methods, in the next section we present our results and analyze them, and finally we conclude.

\section{Method}

The method we use ${ }^{[9]}$ is a straightforward generalization to three dimensions of the technique of Derrida and Vannimenus ${ }^{[10]}$. We calculate the electrical current on a simple cubic lattice of width $m$ and depth $n$ in the horizontal directions, and of length $L$ vertically, growing from bottom up. Periodic boundary conditions are used in the $y$-direction, defined in fig. 1. Each bond between nearest neighbors is randomly conducting, in bond percolation if the bond is occupied (with probability $p$ ), in site percolation if both neighbors are occupied (each with probability $p$ ), otherwise it is insulating. The top and the bottom planes are two equipotentials (fixed boundary condition). One defines by $I_{i}$ and $V_{i}$ the current and potential at the end of each line $i, i=1$ being the index associated with the top plane. The conductance matrix $\sigma_{i, j}$, given through $I_{i}=\sum_{i=1}^{n \cdot m+1} \sigma_{i, j} V_{j}$, is updated via $\sigma_{i, j}^{\prime}=\sigma_{i, j}-\sigma_{i, \alpha} \sigma_{\alpha, j} r /\left(1+\sigma_{\alpha, \alpha} r\right)$ if a longitudinal bond is added at line $\alpha$ and via $\sigma_{i, j}^{\prime}=\sigma_{i, j}+\left(\delta_{\alpha, j}-\delta_{\beta, j}\right) \cdot\left(\delta_{\alpha, i}-\delta_{\beta, i}\right) / r$ if a transversal bond is added between lines $\alpha$ and $\beta$ where $r$ is the resistance of the bond. In that way the conductance is obtained exactly. A very large $L$ (bar configuration) automatically averages over many configurations leaving no ambiguity over whether one should average the conductivity, the resistivity, or functions of them. This is an advantage over methods that average over many disjoint cubes ${ }^{[3,5,6,10]}$.

Fig. 1 Bar of length $L$, width $m$ and depth $n$. We calculate the conductivity between the two black planes. 
We will consider, as in ${ }^{[9]}$, the cases $m=n-1$ for bond percolation and $m=n$ for site percolation. The conductance of the bar per length is then $\sigma_{1,1} / L$ which in the limit $L \rightarrow \infty$ goes to a value $\sigma_{n}$. The calculation is performed for different linear sizes $n$ at the percolation threshold $p_{c}=0.248812^{[11]}$ for bond percolation and $p_{c}=0.311605^{[11]}$ for site percolation. The critical exponent $t / \nu$ is then extracted by using the finite size scaling:

$$
\sigma_{n}=a n^{-t / \nu}\left(1+b n^{-\omega}\right)
$$

where $a$ and $b$ are non-universal constants and $\omega$ is the (first) universal correction to scaling exponent. Since $\nu$, the correlation length exponent, is known rather precisely $(\nu=0.875 \pm 0.003)^{[11]}$ one can determine $t$.

\section{Results and Analysis}

The values obtained with "Percola" using a CPU time of 20 months are given in table 1 for the case of bond and of site percolation. Percola ${ }^{[7]}$, a 64-bit floating point processor runs at 25 MFlops but is specialized to deal with the algorithm described in the previous section for which it runs faster than a Cray-XMP mono-processor. The lengths of the bars presented in table 1 exceed those calculated before ${ }^{[9]}$ by about a factor of 1000 .

\begin{tabular}{|c|c|c|c|c|c|c|}
\hline$n$ & $\sigma_{n}$ & $\Delta \sigma_{n}\left[\times 10^{-5}\right]$ & $L$ & $\sigma_{n}$ & $\Delta \sigma_{n}\left[\times 10^{-5}\right]$ & $L$ \\
\hline 2 & 0.0943868 & 0.32 & $10^{10}$ & 0.1138264 & 0.26 & $10^{10}$ \\
\hline 3 & 0.0484353 & 0.17 & $10^{10}$ & 0.0583394 & 0.27 & $10^{10}$ \\
\hline 4 & 0.0288459 & 0.10 & $2 \cdot 10^{10}$ & 0.0349430 & 0.23 & $1.52 \cdot 10^{10}$ \\
\hline 5 & 0.0187865 & 0.17 & $2.5 \cdot 10^{9}$ & 0.0228411 & 0.23 & $4.6 \cdot 10^{9}$ \\
\hline 7 & 0.0095380 & 0.20 & $10^{9}$ & 0.0119717 & 0.30 & $10^{9}$ \\
\hline 8 & & & & 0.0091521 & 0.12 & $10^{9}$ \\
\hline 10 & 0.0045248 & 0.62 & $3.5 \cdot 10^{7}$ & 0.0058170 & 0.39 & $10^{8}$ \\
\hline 12 & 0.0030573 & 0.27 & $4 \cdot 10^{7}$ & 0.0039910 & 0.43 & $2.2 \cdot 10^{7}$ \\
\hline 13 & 0.0025709 & 0.45 & $8.8 \cdot 10^{6}$ & & & \\
\hline 14 & 0.0021858 & 0.32 & $2.16 \cdot 10^{7}$ & & & $10^{7}$ \\
\hline 15 & 0.0018839 & 0.57 & $10^{7}$ & 0.0024833 & 1.32 & \\
\hline 16 & 0.0016327 & 0.47 & $2 \cdot 10^{6}$ & & & \\
\hline
\end{tabular}

Table 1 Conductance $\sigma_{n}$ per length for bond (left) and site (right) percolation for different widths $n$ of the bar. We also show the statistical mean square deviation $\Delta \sigma_{n}$ and the length $L$ of the bar for each case.

We analyzed the data with various techniques. Finding that $t / \nu$ which minimizes the squared error for fixed $\omega$ (as done in refs. [2] and [8]) gives $t / \nu=2.15 \pm 0.12$ for the sites and $t / \nu=2.26 \pm 0.06$ for the bonds. In both cases $\omega \approx 1.4$ gave the best fits. Since universality states that these exponents should be the same only the intersection of the error bars, i.e. $t / \nu=2.23 \pm 0.06$, should be relevant.

The data for bond percolation are closer to the asymptotic regime as can be seen from the error bars and the curvature in a plot $\log \left(\sigma_{n} n^{t / \nu}\right)$ against $n^{-\omega}$. Looking for 
bond percolation at successive slopes (local derivative of $\log (\sigma)$ vs $\log (n)$ ) against $n^{-\omega}$ gives the most plausible curve for $\omega$ around 1.4. In that case $t / \nu=2.25 \pm 0.05$.

Keeping $t / \nu$ fixed and finding the $\omega$ which minimizes the error (and number of iterations) in the Runge-Kutta procedure of minimizing the mean square deviation, gives a dependence $t / \nu$ against $\omega$ as shown in fig. 2. For $\omega$ around 1.4 we find $t / \nu=2.26 \pm 0.04$ for bond percolation. Unfortunately, as opposed to the case of the superconductivity exponent ${ }^{[8]}$ the prefactor of the site percolation data has the same sign as for bond percolation. Therefore the curves for site percolation in fig. 2 do not intersect with those of bond percolation which does not allow us to increase the precision by assuming universality and combining the two data sets.

Fig. 2 Leading exponent $t / \nu$ against the first correction exponent $\omega$ for bond percolation omitting all sizes of width less or equal to $n_{l}$, for $n_{l}=2$ (diamonds), 3 (crosses + ), 4 (squares) and 5 (crosses $\mathrm{x}$ ).

\section{Conclusion}

Although we have obtained extremely precise data for the conductance of narrow systems our error bars for the exponents $t / \nu$ extrapolated to infinite sizes are not as small as obtained by other methods ${ }^{[5,6]}$. This is due to the fact that we have seriously taken into account the first corrections to scaling which seems imperative for the case of small systems. As with many simulations of high precision, 
statistical errors are much smaller than the systematic ones. In contrast to the two-dimensional case ${ }^{[2]}$ and the superconducting case in three dimensions ${ }^{[8]}$ the relation between leading and correction exponent is not in opposite sense for site percolation as compared to bond percolation giving us a particularly unlucky situation. We conclude $t / \nu=2.26 \pm 0.04$ consistent with previous values and the Alexander-Orbach conjecture ${ }^{[4]}$.

We thank D. Stauffer for help in the evaluation of the data and advice and for helpful suggestions about the manuscript.

\section{References}

1. D. Stauffer and A. Aharony, Introduction to Percolation Theory, (Taylor and Francis, London, 1994)

2. J.-M. Normand, H.J. Herrmann and M. Hajjar, J. Stat. Phys. 52, 441 (1988)

3. D.J. Frank and C.J. Lobb, J. Phys. Rev. B 37, 302 (1988)

4. S. Alexander and R. Orbach, J. Physique Lett. 43, L625 (1982); J. Kertész, J. Phys. A 16, L471 (1983)

5. D.B. Gingold and C.J. Lobb, Phys. Rev. B 42, 8220 (1990)

6. G.G. Batrouni, A. Hansen and B. Larson, preprint

7. F. Hayot, H.J. Herrmann, J.-M. Normand, P. Farthouat and M. Mur, J. Comp. Phys. 64, 380 (1986); M. Hajjar, Thesis (Orsay, 1987) and J.-M. Normand, ACM Conf. Proc., St Malo, 55 (1988)

8. J.M. Normand and H.J. Herrmann, Int. J. of Mod. Phys. C 1, 207 (1990)

9. B. Derrida, D. Stauffer, H.J. Herrmann and J. Vannimenus, J. Physique Lett. 44, L701 (1983)

10. B. Derrida and J. Vannimenus, J. Phys. A 15, L557 (1982)

11. R.M. Ziff, private communication 